\title{
The using effects of Nettle herb in elevating Hb\&PCV levels
}

\author{
Huda Abid AL-Hameed Jassim *
}

Date of acceptance 13/11/2006

\begin{abstract}
:
A dose of ten grams of the roots and leaves of Nettle (Urtica dioica) dissolved in $(200) \mathrm{ml}$ of boiled water then covered for (10)min. was given to a sample of (15) patients attending to the herbal department of ministry of health complaining of malnutrition and low $\mathrm{Hb}$ (hemoglobin) concentration and PCV(packed cell volume) levels with absence of any other predisposing factors disease inorder to find the effects of these roots and leaves on $\mathrm{Hb}$ and PCV levels for different periods of time in relation to age and sex variations .

The study have shown that this mixture has a high significant effect $(\mathrm{p}<0.001)$ in elevating $(\mathrm{Hb})$ concentration and $\mathrm{PCV}$ levels on those patients according to the differences recorded from the start of the basic period until the end of the experiment and this increase was significantly apparent in the $\left(4^{\text {th }}\right)$ week of the experiment. The relation of the age and sex variation were estimated according to the results of the experiment which showed no significant relationship $(\mathrm{p}<0.005)$.
\end{abstract}

\section{Introduction:}

The plant (Nettle) comes from the family urhcaceae and has about 500 species. It can be found in south and north of aljazeera Western desert, Amadia and Rawandose in north part of Iraq (1). It has dispersed throughout the world from
Europe to Asia, Japan, South Africa and Austral in (3).

The clinical and nutritional content of Nettle includes Choline, Chlorophyll formic acid, Iodine, Magnesium, Potassium, Silicon, Iron, Sodium, Sulfur, iron and vitamins A,B and C .(3-4-5)

Medicinal uses: Nettle is known for it's detoxifying and laxative properties in addition to stimulating metabolism, improving digestion and relieving pain (5).

As a result of containing the Nettle such important elements which are involved in homeostasis of red blood cell (R.B.C.) in addition to the other properties of increasing Iron and folic acid absorption (6); therefore this work is undertaken to study the effect of this herb on $\mathrm{Hb}$ and PCV level instead of different medical drugs which may have different or dangerous side effects.

\section{Material and Method:}

Fifteen patients (10 male, 5 female)were attending to the herbal department of Ministry of health with age ranging between $(25-38)$ years suffering from anemia were given roots and leaves of Nettles instead of medicines. Each patient took (10)gm of Nettle (leaves and roots), dissolved in (200)ml of boiling water and left to cool, then became ready for drinking by the patients three times daily after meals and for (4) weeks duration .

* ASSISTANT PROFF /INSTITUTE OF

MEDICAL TECHNOLOGY/FOUNDTION OF

TCHNICAL EDUCATION 
The ( $\mathrm{Hb}$ and PCV) levels were recorded before the start of the treatment and then estimated weekly until the end of the experiment (appendix A, B).

The method of $\mathrm{Hb}$ was analysed by (cyanomethamoglobin) and the method of PCV was analysed by (Haemtcrit centrifuge 10000 R.P.M). (2-7)

The results were tabulated and a statistical analysis was done to find the significant differences.

All calculations were done under the statistical program of social science (spss) v.10.0 on Pentium (3)

\section{The Results:}

The results have shown that there were too highly significant differences at $\mathrm{p}<0.00$, in the $\mathrm{Hb}$ and $\mathrm{pcv}$ levels with development time period of experiment (table 1, figure 1) and (table 2, figure 2).

Table (1) showed descriptive statistics of differences $\mathrm{Hb}$ levels by different period of time

\begin{tabular}{|c|c|c|c|c|}
\hline $\begin{array}{c}\text { Hb } \\
\text { levels } \\
\text { weekly }\end{array}$ & No. & $\begin{array}{c}\text { Mean } \\
\text { gm/100ml }\end{array}$ & $\begin{array}{c}\text { std. } \\
\text { Deviation }\end{array}$ & comparison \\
\hline Hb0 & 15 & 8.933 & 0.753 & \multirow{2}{*}{ H.S } \\
\hline Hb1 & 15 & 9.700 & 0.751 & \\
\hline Hb2 & 15 & 10.067 & 0.704 & \multirow{3}{*}{$\mathrm{p}<\mathbf{0 . 0 0 0}$} \\
\hline Hb3 & 15 & 10.783 & 0.678 & \\
\hline Hb4 & 15 & 11.233 & 0.678 & \\
\hline
\end{tabular}

Fig (1): showed the Hb. Levels with different period of time

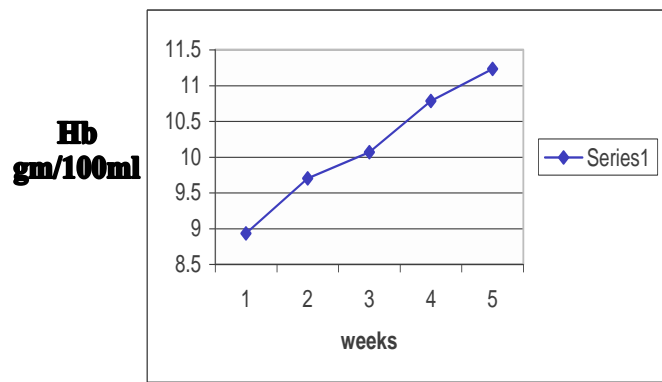

Table (2) showed descriptive statistics of differences pev levels by different period of time

\begin{tabular}{|c|c|c|c|c|}
\hline $\begin{array}{l}\text { pcv levels } \\
\text { weekly }\end{array}$ & No. & $\begin{array}{c}\text { Mean } \\
\%\end{array}$ & $\begin{array}{c}\text { std. } \\
\text { Deviation }\end{array}$ & $\begin{array}{c}\text { c.s } \\
\text { (by-period } \\
\text { t-test) } \\
\text { (p- value) }\end{array}$ \\
\hline Pcv0 & 15 & 25.000 & 0.535 & \multirow{5}{*}{$\begin{array}{c}\text { Hs } \\
0.000\end{array}$} \\
\hline Pcv1 & 15 & 26.330 & 0.585 & \\
\hline Pcv2 & 15 & 26.933 & 0.621 & \\
\hline Pcv3 & 15 & 27.660 & 0.598 & \\
\hline Pcv4 & 15 & 28.420 & 0.648 & \\
\hline
\end{tabular}

Fig (2): showed the PCV. Levels with different period of time

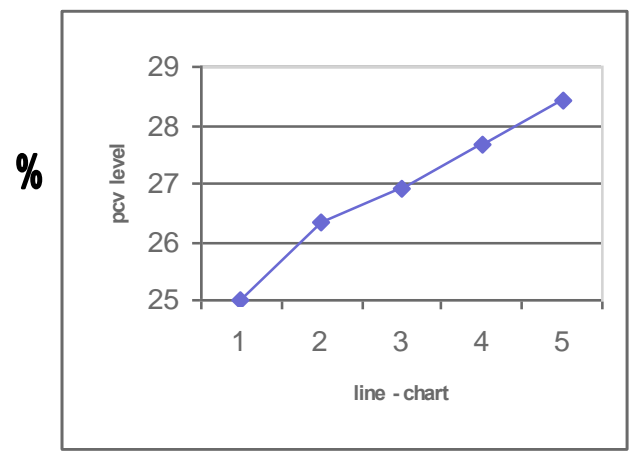

In addition to that pair wise multiple comparisons between different periods of time which shows significant differences of changes between the initial and the final periods of time at $\mathrm{p}<0.05$ whereas it has too highly significance (table 3,4).

Table (3) inferential statistics of different $\mathrm{Hb}$ responses by different period of time

\begin{tabular}{|c|c|c|c|c|c|}
\hline $\begin{array}{c}\text { Time/ } \\
\text { weekly }\end{array}$ & Initial & $\mathbf{1}^{\text {st }}$ & $2^{\text {nd }}$ & $3^{\text {rd }}$ & $4^{\text {th }}$ \\
\hline Initial & - & $\begin{array}{c}\text { HS } \\
(0.024)\end{array}$ & $\begin{array}{c}\text { HS } \\
(0.000)\end{array}$ & $\begin{array}{c}\text { HS } \\
(0.000)\end{array}$ & $\begin{array}{c}\text { HS } \\
(0.000)\end{array}$ \\
\hline $1^{\text {st }}$ & & - & $\begin{array}{c}\text { HS } \\
(0.000)\end{array}$ & $\begin{array}{c}\text { HS } \\
(0.000)\end{array}$ & $\begin{array}{c}\text { HS } \\
(0.000)\end{array}$ \\
\hline $2^{\text {nd }}$ & & & - & $\begin{array}{c}\text { HS } \\
(0.000)\end{array}$ & $\begin{array}{c}\text { HS } \\
(0.000)\end{array}$ \\
\hline $3^{\text {rd }}$ & & & & & $\begin{array}{c}\text { HS } \\
(0.000)\end{array}$ \\
\hline $4^{\text {th }}$ & & & & & - \\
\hline
\end{tabular}


Table (4) descriptive and inferential statistics of different Pcv responses by different period of time.

\begin{tabular}{|c|c|c|c|c|c|}
\hline $\begin{array}{c}\text { Time/ } \\
\text { weekly }\end{array}$ & Initial & $1^{\text {st }}$ & $2^{\text {nd }}$ & $3^{\text {rd }}$ & $4^{\text {th }}$ \\
\hline Initial & - & $\begin{array}{c}\text { HS } \\
(\mathbf{0 . 0 0 0 )}\end{array}$ & $\begin{array}{c}\text { HS } \\
(\mathbf{0 . 0 0 0})\end{array}$ & $\begin{array}{c}\text { HS } \\
(\mathbf{0 . 0 0 0})\end{array}$ & $\begin{array}{c}\text { HS } \\
(\mathbf{0 . 0 0 0})\end{array}$ \\
\hline $1^{\text {st }}$ & & - & $\begin{array}{c}\text { HS } \\
(\mathbf{0 . 0 0 0})\end{array}$ & $\begin{array}{c}\text { HS } \\
(\mathbf{0 . 0 0 0})\end{array}$ & $\begin{array}{c}\text { HS } \\
(\mathbf{0 . 0 0 0})\end{array}$ \\
\hline $2^{\text {nd }}$ & & & - & $\begin{array}{c}\text { HS } \\
(\mathbf{0 . 0 0 1})\end{array}$ & $\begin{array}{c}\text { HS } \\
(\mathbf{0 . 0 0 0})\end{array}$ \\
\hline $3^{\text {rd }}$ & & & & & $\begin{array}{c}\text { HS } \\
(\mathbf{0 . 0 0 0})\end{array}$ \\
\hline $4^{\text {th }}$ & & & & & - \\
\hline
\end{tabular}

Table (5) showed the descriptive statistics of differences which were obtained by initial and final period of times of $\mathrm{Hb}$ factor which showed that the range of differences was (3) $\mathrm{mg} / \mathrm{lit}$.

Table (5) descriptive statistics differences between initial and final period of times of Hb factor

\begin{tabular}{|c|c|c|c|c|c|}
\hline factor & $\mathrm{N}$ & Min. & Max. & Mean & Std.dev. \\
\hline $\begin{array}{c}\text { DHb } \\
\text { ValidN } \\
\text { (List } \\
\text { wise) }\end{array}$ & 15 & 1.5 & 4.5 & 2.367 & 0.812 \\
\hline
\end{tabular}

Table (6) showed some descriptive statistics of differences which were obtain by initial and final period of times of Pcv factor which showed that the range of differences was (6.5).

Table (6) different between initial and final period of time of $\mathrm{PCV}$ factor

\begin{tabular}{|c|c|c|c|c|c|c|}
\hline factor & $\mathrm{N}$ & Range & Min. & Max. & Mean & Std.dev. \\
\hline $\begin{array}{c}\text { DPcv } \\
\text { Valid } \\
\mathbf{N}\end{array}$ & 15 & 5.5 & 1.5 & 7 & 3.353 & 1.320 \\
$\begin{array}{c}\text { (List } \\
\text { wise) }\end{array}$ & 15 & & & & & \\
\hline
\end{tabular}

Table (7, 8) showed the observed frequencies with their percentage within sex differences within total, in addition to that comparison significant of continence coefficient with $\mathrm{P}$ value was obtained which gave a non - significant correlationship between the two factors of table at $p<0.05$.

Table (7) cross tabulation between different levels of different $\mathrm{Hb}$ reading (initial with final period of time)

distributing among both sex

\begin{tabular}{|c|c|c|c|c|c|c|c|}
\hline \multirow{2}{*}{ Sex group } & \multicolumn{5}{|c|}{ Differences of $\mathrm{Hb}$} & \multirow{2}{*}{ Total } & \multirow{2}{*}{$\begin{array}{c}\text { C.C } \\
\text { P.value }\end{array}$} \\
\hline & 1.5 & 2.0 & 2.5 & 3.0 & 4.5 & & \\
\hline $\begin{array}{c}\text { (female) } \\
\text { sex count }\end{array}$ & 3 & - & - & 1 & 1 & 5 & \multirow{12}{*}{$\begin{array}{r}0.604 \\
\text { P }<0.05 \\
\text { (NS) }\end{array}$} \\
\hline $\begin{array}{l}\text { \% within } \\
\text { sex }\end{array}$ & $60 \%$ & - & - & $20 \%$ & $20 \%$ & $\begin{array}{c}100 \\
\%\end{array}$ & \\
\hline $\begin{array}{c}\text { \% within } \\
\text { DHb }\end{array}$ & $75 \%$ & - & - & $\begin{array}{c}33.3 \\
\%\end{array}$ & $\begin{array}{c}100 \\
\%\end{array}$ & $\begin{array}{c}33.3 \\
\%\end{array}$ & \\
\hline$\%$ of total & $20 \%$ & - & - & $6.7 \%$ & $6.7 \%$ & $\begin{array}{c}33.3 \\
\%\end{array}$ & \\
\hline $\begin{array}{c}\text { (Male) } \\
\text { count }\end{array}$ & 1 & 3 & 4 & 2 & - & 10 & \\
\hline $\begin{array}{c}\text { \% within } \\
\text { sex }\end{array}$ & $10 \%$ & $30 \%$ & $40 \%$ & $20 \%$ & - & $\begin{array}{c}100 \\
\%\end{array}$ & \\
\hline $\begin{array}{c}\text { \% within } \\
\text { DHb }\end{array}$ & $25 \%$ & $25 \%$ & $\begin{array}{c}100 \\
\%\end{array}$ & $\begin{array}{c}66.7 \\
\%\end{array}$ & - & $\begin{array}{c}66.7 \\
\%\end{array}$ & \\
\hline$\%$ of total & $6.7 \%$ & $20 \%$ & $\begin{array}{c}26.7 \\
\%\end{array}$ & $\begin{array}{c}13.3 \\
\%\end{array}$ & - & $\begin{array}{c}66.7 \\
\%\end{array}$ & \\
\hline $\begin{array}{c}\text { (Total) } \\
\text { count }\end{array}$ & 4 & 3 & 4 & 3 & 1 & 15 & \\
\hline $\begin{array}{c}\text { \% within } \\
\text { sex }\end{array}$ & $\begin{array}{c}26.7 \\
\%\end{array}$ & $20 \%$ & $\begin{array}{c}26.7 \\
\%\end{array}$ & $20 \%$ & $6.7 \%$ & $\begin{array}{c}100 \\
\%\end{array}$ & \\
\hline $\begin{array}{c}\text { \% within } \\
\text { DHb }\end{array}$ & $\begin{array}{c}100 \\
\%\end{array}$ & $\begin{array}{c}100 \\
\%\end{array}$ & $\begin{array}{c}100 \\
\%\end{array}$ & $\begin{array}{c}100 \\
\%\end{array}$ & $\begin{array}{c}100 \\
\%\end{array}$ & $\begin{array}{c}100 \\
\%\end{array}$ & \\
\hline$\%$ of total & $\begin{array}{c}26.7 \\
\%\end{array}$ & $20 \%$ & $\begin{array}{c}26.7 \\
\%\end{array}$ & $20 \%$ & $6.7 \%$ & $\begin{array}{c}100 \\
\%\end{array}$ & \\
\hline
\end{tabular}

Table (8) cross tabulation between different levels of different $P C V$ reading

\begin{tabular}{|c|c|c|c|c|c|}
\hline \multirow{2}{*}{ Sex group } & \multicolumn{3}{|c|}{ Differences of Pcvs } & \multirow{2}{*}{ Total } & \multirow{2}{*}{$\begin{array}{c}\text { C.C } \\
\text { P.value }\end{array}$} \\
\hline & 1 & 2 & 3 & & \\
\hline $\begin{array}{c}\text { (female) sex } \\
\text { count }\end{array}$ & 3 & 2 & - & 5 & \multirow{12}{*}{$\begin{array}{r}0.232 \\
P<0.05 \\
\text { (NS) }\end{array}$} \\
\hline$\%$ within sex & $60 \%$ & $40 \%$ & - & $100 \%$ & \\
\hline $\begin{array}{c}\text { \% within } \\
\text { DPcvs }\end{array}$ & $42.9 \%$ & $28.6 \%$ & - & $33.3 \%$ & \\
\hline$\%$ of total & $20 \%$ & $13.3 \%$ & - & $33.3 \%$ & \\
\hline (Male) count & 4 & 5 & 1 & 10 & \\
\hline \% within sex & $40 \%$ & $50 \%$ & $10 \%$ & $100 \%$ & \\
\hline $\begin{array}{l}\text { \% within } \\
\text { DPcvs }\end{array}$ & $57.1 \%$ & $71.4 \%$ & $100 \%$ & $66.7 \%$ & \\
\hline$\%$ of total & $26.7 \%$ & $33.3 \%$ & $6.7 \%$ & $66.7 \%$ & \\
\hline (Total) count & 7 & 7 & 1 & 15 & \\
\hline$\%$ within sex & $46.7 \%$ & $46.7 \%$ & $6.7 \%$ & $100 \%$ & \\
\hline $\begin{array}{c}\text { \% within } \\
\text { DHb }\end{array}$ & $100 \%$ & $100 \%$ & $100 \%$ & $100 \%$ & \\
\hline$\%$ of total & $46.7 \%$ & $46.7 \%$ & $6.7 \%$ & $100 \%$ & \\
\hline
\end{tabular}




\section{(initial with final period of time)}

Table $(9,10)$ showed the observed frequencies with their percentage within age and differences within total, in addition to that comparison significant of continence coefficient with $p$ value was obtain which gave a non- significant correlationship between the two factors of table at $\mathrm{p}<0.05$.

Table (9) cross tabulation between different levels of different $\mathrm{Hb}$ reading (initial with final period of time) distributing among both age group

\begin{tabular}{|c|c|c|c|c|c|c|c|}
\hline \multirow{2}{*}{$\begin{array}{c}\text { Age } \\
\text { group }\end{array}$} & \multicolumn{5}{|c|}{ Differences of $\mathbf{H b}$} & \multirow{2}{*}{ Total } & \multirow{2}{*}{$\begin{array}{c}\text { C.C } \\
\text { P.value }\end{array}$} \\
\hline & 1.5 & 2.0 & 2.5 & 3.0 & 4.5 & & \\
\hline $\begin{array}{l}\text { Ages } \\
\text { (1) } \\
\text { count }\end{array}$ & 1 & 3 & 2 & 1 & - & 7 & \multirow{12}{*}{$\begin{array}{r}0.511 \\
P<0.05 \\
\text { (NS) }\end{array}$} \\
\hline $\begin{array}{c}\% \\
\text { within } \\
\text { ages }\end{array}$ & $14.3 \%$ & $42.9 \%$ & $\begin{array}{c}28.6 \\
\%\end{array}$ & $\begin{array}{c}14.3 \\
\%\end{array}$ & - & $100 \%$ & \\
\hline $\begin{array}{c}\% \\
\text { within } \\
\text { DHb }\end{array}$ & $25 \%$ & $100 \%$ & $\mathbf{5 0 \%}$ & $\begin{array}{l}50 \\
\%\end{array}$ & $33.3 \%$ & $46 \%$ & \\
\hline $\begin{array}{l}\% \text { of } \\
\text { total }\end{array}$ & $6.7 \%$ & $20 \%$ & $\begin{array}{c}13.3 \\
\%\end{array}$ & $\begin{array}{l}6.7 \\
\%\end{array}$ & - & $46 \%$ & \\
\hline $\begin{array}{c}(2) \\
\text { count }\end{array}$ & 3 & - & 2 & 2 & 1 & 8 & \\
\hline $\begin{array}{c}\% \% \\
\text { within } \\
\text { ages }\end{array}$ & $37.5 \%$ & - & $25 \%$ & $\begin{array}{l}25 \\
\%\end{array}$ & $12.5 \%$ & $100 \%$ & \\
\hline $\begin{array}{c}\% \\
\text { within } \\
\text { DHb }\end{array}$ & $75 \%$ & - & $50 \%$ & $\begin{array}{c}66.7 \\
\%\end{array}$ & $100 \%$ & $53.3 \%$ & \\
\hline $\begin{array}{l}\% \text { of } \\
\text { total }\end{array}$ & $20 \%$ & - & $\begin{array}{c}13.3 \\
\%\end{array}$ & $\begin{array}{c}13.3 \\
\%\end{array}$ & $6.7 \%$ & $53.3 \%$ & \\
\hline $\begin{array}{l}\text { (Total) } \\
\text { count }\end{array}$ & 4 & 3 & 4 & 3 & 1 & 15 & \\
\hline $\begin{array}{c}\% \\
\text { within } \\
\text { ages }\end{array}$ & $26.7 \%$ & $20 \%$ & $\begin{array}{c}26.7 \\
\%\end{array}$ & $\begin{array}{l}20 \\
\%\end{array}$ & $6.7 \%$ & $100 \%$ & \\
\hline $\begin{array}{c}\% \\
\text { within } \\
\text { DHb }\end{array}$ & $100 \%$ & $100 \%$ & $\begin{array}{c}100 \\
\%\end{array}$ & $\begin{array}{c}100 \\
\%\end{array}$ & $100 \%$ & $100 \%$ & \\
\hline $\begin{array}{l}\% \text { of } \\
\text { total }\end{array}$ & $26.7 \%$ & $20 \%$ & $\begin{array}{c}26.7 \\
\%\end{array}$ & $\begin{array}{l}20 \\
\%\end{array}$ & $6.7 \%$ & $100 \%$ & \\
\hline
\end{tabular}

Table (10) cross tabulation between different levels of different $\mathrm{PCVs}$ reading (initial with final period of time) distributing

\begin{tabular}{|c|c|c|c|c|c|}
\hline \multirow{2}{*}{$\begin{array}{c}\text { Age } \\
\text { group }\end{array}$} & \multicolumn{3}{|c|}{ Differences of Pcvs } & \multirow{2}{*}{ Total } & \multirow{2}{*}{$\begin{array}{c}\text { C.C } \\
\text { P.value }\end{array}$} \\
\hline & 1 & 2 & 3 & & \\
\hline $\begin{array}{c}\text { Ages (1) } \\
\text { count }\end{array}$ & 3 & 3 & 1 & 7 & \multirow{12}{*}{$\begin{array}{c}0.275 \\
\text { P<0.05 } \\
\text { (NS) }\end{array}$} \\
\hline $\begin{array}{c}\% \text { within } \\
\text { ages }\end{array}$ & $42.9 \%$ & $42.9 \%$ & $14.3 \%$ & $100 \%$ & \\
\hline $\begin{array}{c}\text { \% within } \\
\text { DPcvs }\end{array}$ & $42.9 \%$ & $42.9 \%$ & $100 \%$ & $46.7 \%$ & \\
\hline \% of total & $20 \%$ & $20 \%$ & $6.7 \%$ & $46.7 \%$ & \\
\hline (2) count & 4 & 4 & - & 8 & \\
\hline $\begin{array}{c}\begin{array}{c}\% \text { within } \\
\text { ages }\end{array} \\
\end{array}$ & $50 \%$ & $50 \%$ & - & $100 \%$ & \\
\hline $\begin{array}{c}\text { \% within } \\
\text { DPcvs }\end{array}$ & $57.1 \%$ & $57.1 \%$ & - & $53.3 \%$ & \\
\hline \% of total & $26.7 \%$ & $26.7 \%$ & - & $53.3 \%$ & \\
\hline $\begin{array}{c}\text { (Total) } \\
\text { count }\end{array}$ & 7 & 7 & 1 & 15 & \\
\hline $\begin{array}{c}\% \text { within } \\
\text { ages }\end{array}$ & $46.7 \%$ & $46.7 \%$ & $6.7 \%$ & $100 \%$ & \\
\hline $\begin{array}{c}\text { \% within } \\
\text { DPcvs }\end{array}$ & $100 \%$ & $100 \%$ & $100 \%$ & $100 \%$ & \\
\hline$\%$ of total & $46.7 \%$ & $46.7 \%$ & $6.7 \%$ & $100 \%$ & \\
\hline
\end{tabular}

distributing among both sex among both age group

\section{Discussion:}

From the descriptive statistics mean, standard deviation as whole multiple comparison of different period of times for $\mathrm{Hb}$ and Pcv factors, (Table1, 2), The results have shown that there were too highly significant differences at $\mathrm{P}<0.000$, whereas the figure of line chart represents the high changes which were obtained by the effect of the suggested Nettle .The result shows clearly the increase of the $\mathrm{Hb}$ and Pcv levels with the developing time period of the experiment (4-5) . In addition to that pair wise multiple comparison between different periods of time shows significant differences of change between initial and the first period of time at 0.05 whereas highly significant difference at $\mathrm{p}<0.000$, at the others contrast pairs comparison were obtained. These results gave an attention to put an extreme period of time (4) weeks as a final of this study, because the over dose may cause damage in hepatic tissues (8). The descriptive statistics of differences which were obtained by initial and final period of times of $\mathrm{Hb}$ and Pcv factors have shown that the range of differences was (3)mg/lit for $\mathrm{Hb}$ level and 6.5 for Pcv gave highly grade of changes between sample individual patients(58).table(5,6). From the observed frequencies with their percentage within sex and differences which were mentioned by the previous table and within total, in addition to that a comparison significance of continence coefficient with $\mathrm{P}$ value were obtained and gave a non-significant correlationship parameter was approximately accepted(9-10).(Table $7,8)$. The observed frequencies with their percentage within age and differences which were mentioned by the previous 
table and within total. In addition to that Coefficient with(P)value was obtained which gave a non- significant correlationship between the factor of table at $p<0.05$ which revealed to cause effect of small sample size whereas the result correlationship parameter was approximately accepted (Table9,10).

According to the results and discussion we conclude the following:

1. There are too highly significant differences at $\mathrm{p}<0.001$.

2. There was a clear increase of the $\mathrm{Hb}$ and Pcv levels during the comparison significant of continence developing time of period of the experiment.

3. The range of differences was (3) $\mathrm{mg} / \mathrm{lit}$ and 6.5 , which deals with highly grade of changes between sample individual patient.

4. There was a non-significant correlationship between the observed frequencies with their percentage within sex or ages at $\mathrm{p}<0.05$ which revealed to causes effect of small size.

\section{Appendix (A)}

Original observation for $\mathrm{Hb}$ repeated measurement according to the different period of time

\begin{tabular}{|c|c|c|c|c|c|c|c|c|c|}
\hline \multirow{2}{*}{$\begin{array}{c}\text { No of } \\
\text { patient }\end{array}$} & \multirow{2}{*}{ Age } & \multirow{2}{*}{$\begin{array}{c}\text { Age } \\
\text { by }\end{array}$} & \multirow{2}{*}{ Sex } & \multicolumn{5}{|c|}{ PCV level (weekly) } & \multirow{2}{*}{ DPCV } \\
\cline { 5 - 8 } & & score & & Pcv0 & Pcv1 & Pcv2 & Pcv3 & Pcv4 & \\
\hline 1 & 15 & 1 & M & 23 & 23 & 24 & 26 & 27 & 4 \\
\hline 2 & 15 & 1 & M & 25 & 26 & 26 & 27 & 28 & 3.5 \\
\hline 3 & 16 & 1 & M & 26 & 27 & 28 & 28 & 28 & 2.5 \\
\hline 4 & 20 & 1 & M & 26 & 27 & 28 & 28 & 33 & 7 \\
\hline 5 & 20 & 1 & M & 29 & 31 & 32 & 33 & 33 & 4 \\
\hline 6 & 22 & 1 & M & 30 & 31 & 32 & 33 & 33 & 3 \\
\hline 7 & 23 & 1 & M & 24 & 25 & 26 & 26 & 26 & 1.5 \\
\hline 8 & 25 & 2 & M & 24 & 25 & 26 & 26 & 26 & 2.5 \\
\hline 9 & 28 & 2 & M & 24 & 26 & 27 & 27 & 28 & 4.5 \\
\hline 10 & 28 & 2 & M & 25 & 26 & 26 & 27 & 28 & 3.5 \\
\hline 11 & 28 & 2 & F & 25 & 26 & 26 & 27 & 27 & 2 \\
\hline 12 & 30 & 2 & F & 24 & 25 & 26 & 26 & 26 & 2.5 \\
\hline 13 & 30 & 2 & F & 24 & 25 & 25 & 26 & 26 & 2.4 \\
\hline 14 & 34 & 2 & F & 23 & 24 & 25 & 25 & 26 & 3.4 \\
\hline 15 & 38 & 2 & F & 23 & 24 & 25 & 26 & 27 & 4 \\
\hline
\end{tabular}

Appendix (B)

Original observation for PCV repeated measurement according to the different period of time

\begin{tabular}{|c|c|c|c|c|c|c|c|c|c|}
\hline \multirow{2}{*}{ No of patient } & \multirow{2}{*}{ Age } & \multirow{2}{*}{$\begin{array}{l}\text { Age by } \\
\text { score }\end{array}$} & \multirow{2}{*}{$\operatorname{sex}$} & \multicolumn{5}{|c|}{ Hb level (weekly) } & \multirow{2}{*}{ DHb } \\
\hline & & & & Hbo & Hb1 & $\mathrm{Hb2}$ & Hb3 & Hb4 & \\
\hline 1 & 15 & 1 & M & 8 & 8 & 8.5 & 9 & 9.5 & 1.5 \\
\hline 2 & 15 & 1 & $\mathbf{M}$ & 9 & 10 & 10 & 11 & 11 & 2 \\
\hline 3 & 16 & 1 & $\mathbf{M}$ & 9 & 10 & 10 & 11 & 11 & 2 \\
\hline 4 & 20 & 1 & M & 9.5 & 9.5 & 10 & 10 & 11 & 1.5 \\
\hline 5 & 20 & 1 & $\mathbf{M}$ & 10 & 11 & 11 & 11 & 12 & 2 \\
\hline 6 & 22 & 1 & $\mathbf{M}$ & 9 & 9.5 & 10 & 10 & 11 & 2 \\
\hline 7 & 23 & 1 & M & 8 & 9 & 9.5 & 10 & 11 & 3 \\
\hline 8 & 25 & 2 & $\mathbf{M}$ & 9.5 & 10 & 10 & 11 & 12 & 2.5 \\
\hline 9 & 28 & 2 & $\mathbf{M}$ & 9 & 9.5 & 10 & 11 & 11 & 2.5 \\
\hline 10 & 28 & 2 & $\mathbf{M}$ & 8 & 9.5 & 15 & 10 & 11 & 3 \\
\hline 11 & 28 & 2 & $\mathbf{F}$ & 9.5 & 9.5 & 9.5 & 10 & 11 & 1.5 \\
\hline 12 & 30 & 2 & $\mathbf{F}$ & 8 & 9 & 9.5 & 10 & 11 & 3 \\
\hline 13 & 30 & 2 & $\mathbf{F}$ & 8 & 10 & 11 & 12 & 12 & 4 \\
\hline 14 & 34 & 2 & $\mathbf{F}$ & 9.5 & 10 & 10 & 11 & 11 & 1.5 \\
\hline 15 & 38 & 2 & $\mathbf{F}$ & 10 & 11 & 10 & 11 & 11 & 1.5 \\
\hline
\end{tabular}




\title{
References:
}

1. رويحة أمين(1990)،التداوي بالاعشاب، علوم

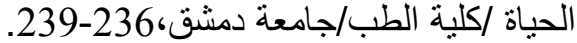

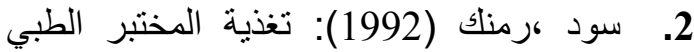

(طر ائق وتفسير ات )ترجمة :صالح خميس حيدر

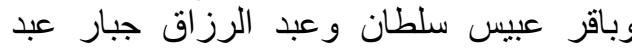

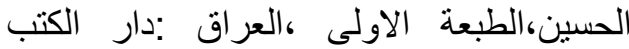

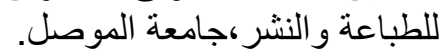

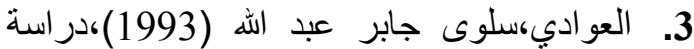

بعض الاعشاب الطبيةفي العراق على على البكتريا

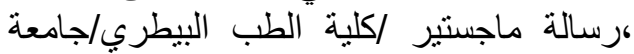

$$
\text { بغداد. }
$$

4. Hirano, T. Homma M,Oka K. 1994.

Effects of stinging nettle root extract and their steroidal component on the $\mathrm{Na}+\mathrm{K}+-$ ATpase of the benign prostatic hyperplasia Planta med. $\quad 60: 30$ 33.

5. Blumenthal ,M. Busse, WR, Goldberg

A . 1998. (Eds).the complete commission E monographs: therapeutic guide to Herbal medicines .Boston,MA: integrative medicine communication :216-217.
6. W.B sounder's co. Philadelphia. 1977. Dorland's pocket medical dictionary, 22nd, pp, 37 -38.

7. Dasia ,J.V. (1989). Practical hematology, London: company.ltd, p.12.

8. Blach, JF and P.A. 1977. Prescription for nutrition heal,a very publishing group .Garden city park ,NY, pp,128 -129.

9. Cao - CJ; Eldefrawi - ME; Eldefrawi $\quad-\mathrm{AT}$; Burnett $-\mathrm{JW}$; Mioduszewski - R.J; Monking DE ;Valdes -JJ. 1998.Toxicity effect of sea Nettle toxin to human nepatoeytes and the protective effect of phosphorylating and alkylating agent .Toxicon 36 (2):264 - 268.

10. Randall, C ,Meethan K ,Randall H Dobbs F. 1999.Nettle stinging of Urtica dioica for joint pain - an exploratory study of this complementary therapy .Compl Ther Med:7:126 -131.

\section{تأثير استخدام عشبة القراص في مستوى خضاب الام وكريات الدم الضغوطة

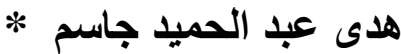

\section{* أستاذ مساعد المعهد الطبي التقني /هيئة التعليم التقني}

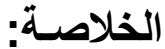

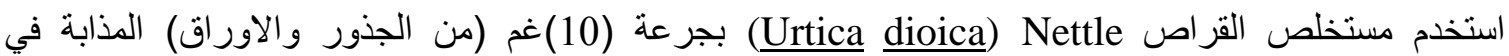
(200)ملي لتر ماء مغلي تركت مغطاة لمدة (10)دقائق .

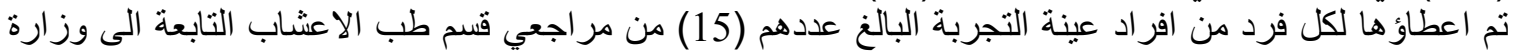

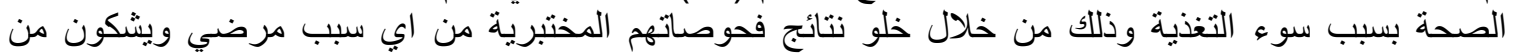

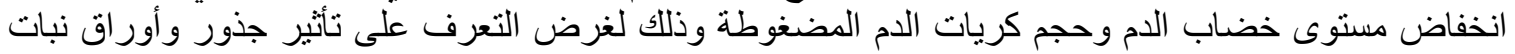

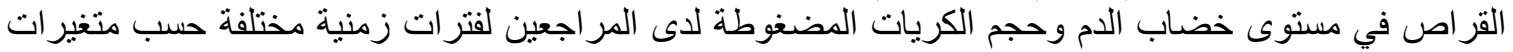

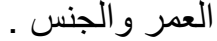
اظهرت الدراسة ان لهذا الخليط تأثير معنوي عال عند (P>0.001) في زيادة مستوى خضاب الدم وحجم كريات

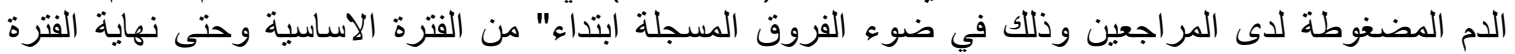

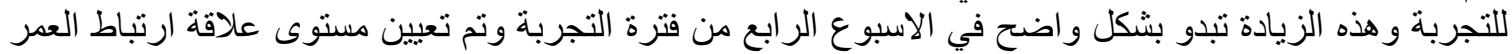
والجنس مع نتائج المستويات التجريبية ايضا حيث اسفرت عن عدم تحقق العلاقة المعنوية عند مستوى الدئلالة
\end{abstract}

. $(\mathrm{P}<0.05)$ 